\section{Brilliant pebbles won't do}

SIR-In their recent Commentary!, G. Canavan and E. Teller conclude that singlet space-based interceptors (SBI) are ". . . effective in the near-term and midterm ... ." and that they can be made adequately survivable against achievable Soviet reactions by "hardening, evasion, and decoy in combination ....". These conclusions stem from the constraints imposed by the authors on the Soviet response. Unaccountably, they assume the Soviets will be able to reduce missile burn time $T$ only to 300 seconds or so. But the Soviets already deploy SS-25 missiles with $T=180$ seconds, corresponding to an availability of $1.1 \%$ for the SBI. Subtracting the SBI "release time" of 50-100 seconds would further reduce the effectiveness of SBI, according to Lt Gen. George L. Monahan's formal response to the US Senate Appropriations Committee on the SDI programme.

Ten warheads deployed on silo-based single-warhead SS-25s within a region a few hundred kilometres or less across would require $10 / 1.1 \%=900$ brilliant pebbles to be able to assign even a single interceptor to each missile. As few as 1,000 ICBM warheads would thus need 90,000 brilliant pebbles - a clear demonstration that the defence is not cost-effective at the margin.

The Senate Appropriations Committee went on to ask whether the SDI phase I architecture would be capable of destroying more than a few SS-25s in the boost phase. Monahan answered "No. The phase I SDS (Strategic Defense System) does not provide continuous coverage of the SS-25 deployment region given the available time (SS-25 burn time minus surveillance/command and control timeline)."

If the burn time of a new solid-fuel missile were reduced to $T=100$ seconds, there would be no intercepts at all. The launch weight of a booster designed to deploy its single warhead in 100 seconds would be about $5 \%$ above that of a missile of normal burn time ${ }^{2}$.

Canavan and Teller dismiss an even more important and less costly countermeasure: "Attrition during peacetime . .

While anti-satellites would be effective in that role, their use might be viewed as an attack, so the main danger in this case would probably come from ground-based lasers." But the Soviets already launch hundreds of test missiles a year without our viewing them as an "attack". A deployment of SBI could provoke the building of a similar number of Soviet small ASAT weapons, with 40-kilogram homing warheads. Only about 3.5 kilometres per second need be supplied by a two-stage rocket to lift the ASAT to 400kilometre altitude at precisely the time an

\section{Identity crisis}

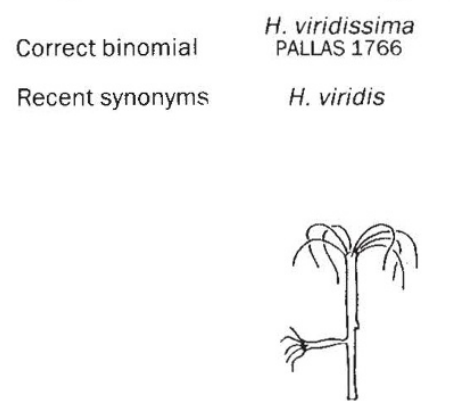

\section{Colour}

Number of tentacles on young bud Length of body

Length of tentacles

Stenoteles

(

$$
\text { re }
$$
relative to body

SBI is expected at the intercept point. Launching such a small, slow rocket in Kazakhstan could not be regarded as an attack on the United States; it could not reach us, but it could reach our space weapons overflying the Soviet Union.

The key points in making the ASAT clearly cheaper ${ }^{3.4}$ and simpler than the brilliant pebbles are that the rocket mass required to launch a 40-kilogram ASAT homing head to intercept altitude is a factor of seven smaller than that required to launch into orbit a 40-kilogram SBI (bearing a 5-kilogram homing head), and the much easier task of an ASAT performing its job within full view of ground-based radar and optical sensors, designators and compound intelligence. This ground-based support would allow the ASAT to identify and destroy manoeuverable, decoyed SBI, or (equally effective) to provoke them to manoeuvere and dispense decoys

SIR-Hydra are used frequently in research and teaching ${ }^{1.2}$, but identifying them is extremely difficult because names have been used inconsistently. Recent work has clarified the names and identities of the five species described in Europe ${ }^{3-5}$. The nematocysts ("stingers") provide the most certain means of identification, and the morphological characters of the body of hydra, as shown in the figure, provide H. Oligactis
PALLAS 1766 PALLAS 1766

so that they will be vulnerable weeks or months later.

If helpful, intercepts could be timed so that the intercept takes place in daylight while an observation point on the ground is in the dark. With a phase-I SDI deployment of 4,000 SBI, an SBI would fly within 50 kilometres of the vertical over the ASAT launch site about 20 times an hour, so there are many opportunities for intercept. A deployment of 40-kilogram brilliant pebbles could be defeated at much lower cost and thus would impair rather than add to US security. RICHARD L. GARWIN

IBM Research Division,

Thomas J. Watson Research Center,

PO Box 218,

Yorktown Heights,

New York 10598, USA

1. Canavan, G. \& Teller, E. Nature 344, 699-704 (1990)

2. Bloembergen, N. \& Patel, C. K. Rev. Mod. Phys, 59 , No. 3 Part II S30-32 (1987)

3. Speed, R. D. Lawrence Livermore National Laboratory Report UCRL-ID-103669 (1990)

4. Garwin, R. L. Nature 344, 301-302 (1990)

simple guides for identifying the species $H$. viridissima is immediately identifiable by its green colour, and $H$. oligactis's identity is revealed by two precocious tentacles on young buds. Identification of the other three species on the basis of morphological criteria is more difficult because the lengths of the tentacles and body vary with culture conditions.

One reason for confusion among names is that the species of hydra most widely used in laboratory research has been

\title{
$\begin{array}{ccc}\text { H. vulgaris } & \text { H. circumcincta } & \text { H. oxycnida } \\ \text { PALLAS } 1766 & \text { SCHULZE 1914 } & \text { SCHULZE 1914 }\end{array}$
}

H. attenuata
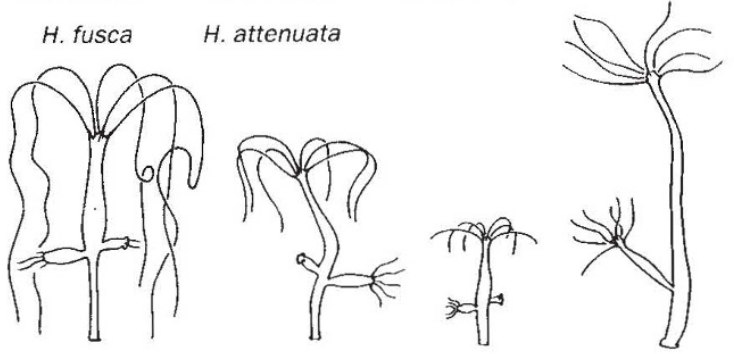

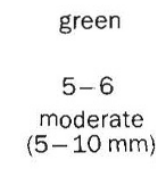

$1 / 2-1$

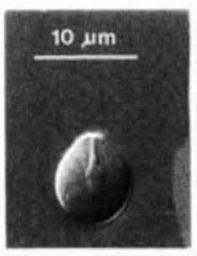

Holotrichous isorhizas

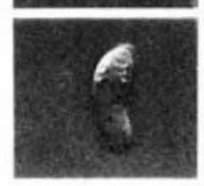

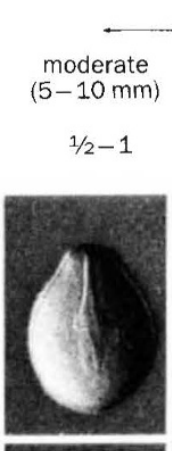

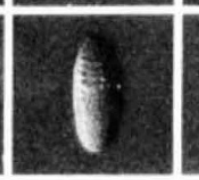

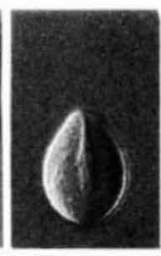

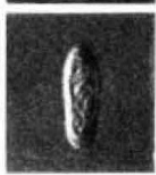

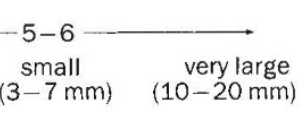

$(3-7 \mathrm{~mm})$

$1 / 2-1$
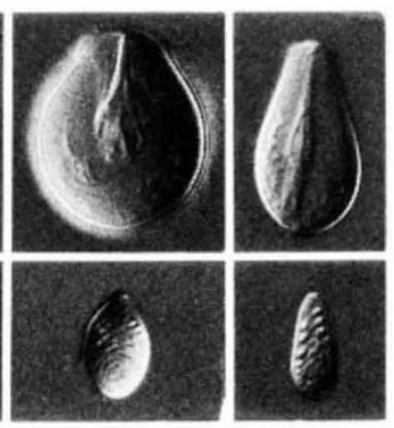